\title{
Method of Polychromatic Hilbert Diagnostics of Phase and Temperature Perturbations of Axisymmetric Flames
}

\author{
Vitaly Arbuzov 1,3, Eduard Arbuzov ${ }^{1,2,3}$, Yuri Dubnishchev ${ }^{1,3}$, Olga Zolotukhina ${ }^{1,3}$ and \\ Vladimir Lukashov ${ }^{1}$ \\ 1 Kutateladze Institute of Thermophysics of the Siberian Branch of the Russian Academy of Sciences, 1 \\ Academician Lavrentiev Avenue, Novosibirsk, 630090, Russia \\ ${ }^{2}$ Sobolev Institute of Mathematics of the Siberian Branch of the Russian Academy of Sciences, 4 Academician \\ Koptyug Avenue, Novosibirsk, 630090, Russia \\ ${ }^{3}$ Novosibirsk State Technical University, 20 Karl Marx Avenue, Novosibirsk, 630073, Russia
}

\begin{abstract}
The work is aimed at solving the scientific and practical problem of non-disturbing diagnostics of the phase and temperature fields of reacting jets and flames. On the example of an axisymmetric hydrogen-diffusion flame and a hot air flow from a candle flame, a method was developed that is adequate to the problem being solved, based on Hilbert polychromatic visualization of phase optical density fields, measuring the temperature profile in selected areas of the medium under study, pixel-by-pixel processing of RAW images recorded by a photographic matrix in RGB channels. The visualized Hilbert structures carry information about the phase optical density perturbations induced by the temperature field. The phase structure of the probing light field in the axial symmetry approximation of the flame under investigation is analyzed using the Abel transform. Iterative selection of radial temperature profiles, adapted Bezier curves, is performed with the subsequent calculation of the spatial structure of the refractive index and phase function. The reconstruction of the temperature field by the example of the study of a hydrogen-air flame is carried out taking into account the diversity of the partial optical properties of the gas mixture in a model consistent with the Gladstone-Dale dispersion formula. The influence of disturbances in the air surrounding the flame on its axial symmetry is discussed. The criterion for the reliability of the research results is a comparison of the hilbertograms obtained in the experiment and the hilbertograms reconstructed from phase structures induced by temperature fields.
\end{abstract}

\section{Keywords}

Optical diagnostics of a flame, hydrogen-air diffusion flame, Hilbert optics, hilbertogram

\section{Introduction}

To date, the focus of scientific research is the problems associated with the stabilization of combustion, the features of heat release in the reacting stream and the formation of harmful emissions in combustion products [1]. An adequate solution to these problems is associated with the development of optical diagnostics of reacting jets and flames with obtaining information on the thermodynamic and structural parameters of the medium under study. Modern methods of spectroscopy LIF and RAMAN provide data on the temperature and chemical composition of the flame [2]. At the same time, methods based on diagnostics of the optical phase density fields of the plume remain in demand. In combination with classical methods of visualization of flows [3] using optical tomography [4], such approaches have great potential.

GraphiCon 2021: 31sh International Conference on Computer Graphics and Vision, September 27-30, 2021, Nizhny Novgorod, Russia EMAIL: arbuzov@itp.nsc.ru (V.A. Arbuzov); arbuzov@math.nsc.ru (E.V. Arbuzov); dubnistchev@itp.nsc.ru (Yu.N. Dubnishchev); melexina-olga17@yandex.ru (O.S. Zolotukhina); luka@itp.nsc.ru (V.V. Lukashov)

ORCID: 0000-0003-2404-526X (V.A. Arbuzov); 0000-0001-9488-8650 (E.V. Arbuzov); 0000-0001-7874-039X (Yu.N. Dubnishchev); 00000003-3486-4459 (O.S. Zolotukhina); 0000-0001-8178-7607 (V.V. Lukashov)

(c) (i) 2021 Copyright for this paper by its authors.

Use permitted under Creative Commons License Attribution 4.0 International (CC BY 4.0).

CEUR Workshop Proceedings (CEUR-WS.org) 
The article [5] describes a method for assessing the temperature distribution in an axisymmetric flame using high-contrast stereoscopic photography. Spectral reconstruction of temperature fields using pyrometry of color ratios and interferometric tomography is reported [6]. Reference [7] discusses the principles of optical testing of transparent objects using the Talbot images method to diagnostic of flames. An example of adapted to the problems of flame research is optical diagnostics based on the methods of Hilbert optics and interferometry in combination with pixel-by-pixel processing of the dynamic structure of visualized phase structures induced by temperature fields [8].

The aim of this work is to develop and adapt methods of polychromatic Hilbert optics for diagnosing the optical density fields of phases and flame temperature with the reconstruction of the spatial phase and temperature structure.

\section{Hilbert visualization of the phase structure of the flame}

The diagnostic complex is based on the IAB-451 shadow device [9] with modified modules for optical filtering, light emitter, registration and information processing. The scheme of the complex for optical Hilbert diagnostics of an axisymmetric flame is similar to that given in [10].

The lighting module consists of a light source - an RGB LED with operating wavelengths $\lambda_{1}=636$ $\mathrm{nm}, \lambda_{2}=537 \mathrm{~nm}$ and $\lambda_{3}=466 \mathrm{~nm}$, a collimator lens and a slit diaphragm located in the front Fourier plane of the lens, which forms a luminous flux. The Fourier spectrum of phase perturbations induced in the probing light field by a flame is localized in the frequency plane of the Fourier lens, where the Hilbert quadrant filter is located. Depending on the spectral characteristics of the light source, Hilbert filtering converts the light field into an optical analytical signal [11] recorded by a digital video camera connected to a computer [12].

In figure 1 shows the Hilbert images of a hydrogen-air flame obtained by simultaneous shooting at three different wavelengths of the radiation source $-\lambda_{1}=636 \mathrm{~nm}, \lambda_{2}=537 \mathrm{~nm}$ and $\lambda_{3}=466 \mathrm{~nm}$. The probe radiation was detected by the matrix of digital camera "Canon EOS 650D" with dimensions of $22,3 \times 14,9 \mathrm{~mm}$ and a number of active pixels are $18 \mathrm{Mpx}$. The frames were taken at a shutter speed of $1 / 60 \mathrm{~s}$ and with a resolution of $5184 \times 3456 \mathrm{px}$. The fuel mixture $\mathrm{H}_{2} / \mathrm{N}_{2}$ was fed vertically into stationary air through a pipe with an inner diameter of $d=5 \mathrm{~mm}$ and a length of $500 \mathrm{~mm}$. The tube material is stainless steel. The experiments were carried out in the range of Reynolds numbers $R e=1000 \ldots 2000$. At these parameters, a laminar Poiseuille flow was established in the tube. The composition and consumption of the fuel mixture of hydrogen with nitrogen was set using a software generator of calibration gas mixtures UFPGS-2.
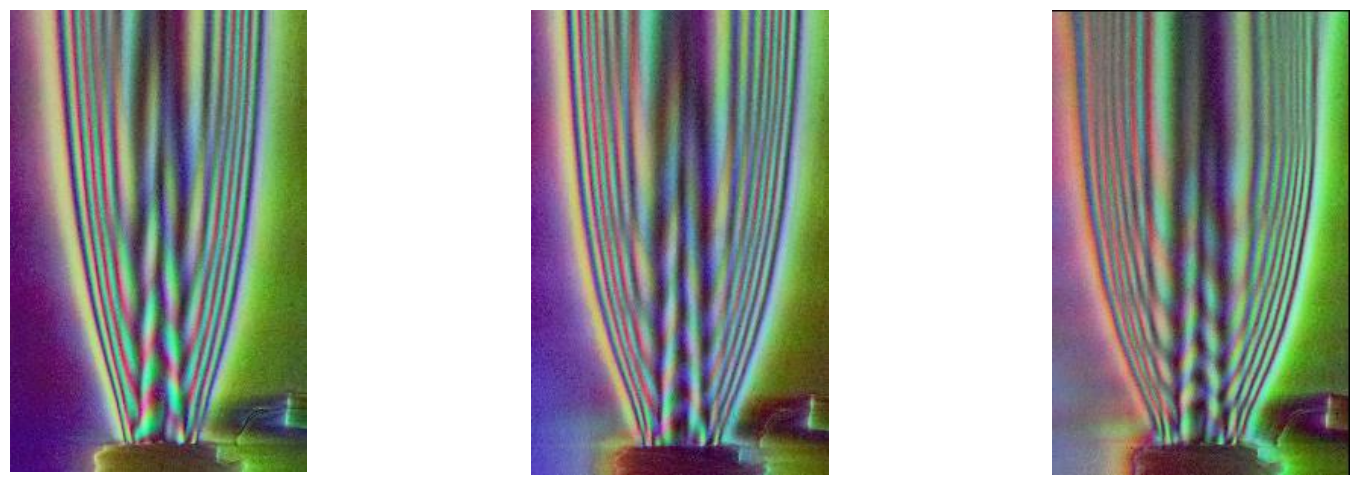

Figure 1: Hilbert images of a diffuse hydrogen-air flame diffusion obtained by simultaneous shooting at three different wavelengths of the radiation source $-\lambda_{1}=636 \mathrm{~nm}, \lambda_{2}=537 \mathrm{~nm}$ and $\lambda_{3}=466 \mathrm{~nm}$

The phase structure of the probing light field in the study of an axisymmetric flame is determined through the Abel integral as

$$
\Delta \psi(r, y)=2 k \int_{x}^{R}\left[n(r, y)-n_{0}\right] \frac{r d r}{\sqrt{r^{2}-x^{2}}},
$$

where $r^{2}=x^{2}+z^{2} ; k=2 \pi / \lambda$ is the wavenumber; $R$ is the radius of the burner section; $n(r, y)$ is the refractive index as a function of the distance $\mathrm{r}$ from the center of the section selected in the $y=$ const plane; $n_{0}$ is the refractive index of the medium undisturbed by the flame (figure 2 ). The $z$-axis is set by 
the direction of the probe light beam. Coordinates $z_{1}, z_{2}$ limit the size of the flame section in the direction of the probe beam, and $y$ indicates the position of the section along the flame axis.

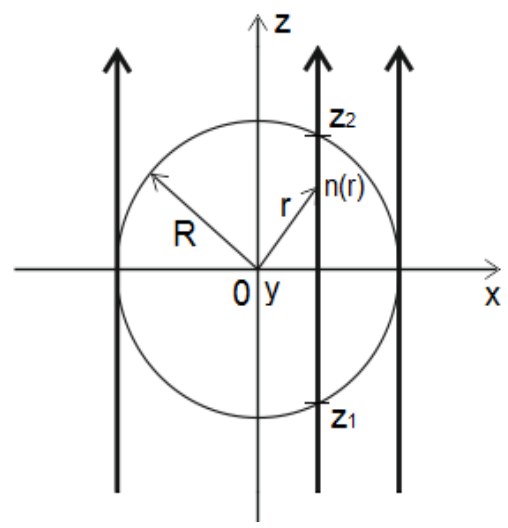

Light beam

Figure 2: Section of the investigated axisymmetric object in the $y=$ const plane

\section{Reconstruction of the temperature field of the air flow induced by the flame of a candle}

When studying the flow induced by the flame of a candle, the axisymmetric distributions of the refractive index $n(r, y)$ obtained from the Abel equations determine the temperature fields $T(r, y)$ in the selected flame section using the Gladstone-Dale equation [13]:

$$
T(r, y)=\left[\frac{n_{0}-1}{n(r, y)-1}\right] T_{0},
$$

where $T_{0}$ is the temperature and $n_{0}$ is the refractive index of the undisturbed medium surrounding the flame.

In figure 3 shows RGB Hilbert images of heated airflow from a candle flame, chromatically selected on RGB channels.

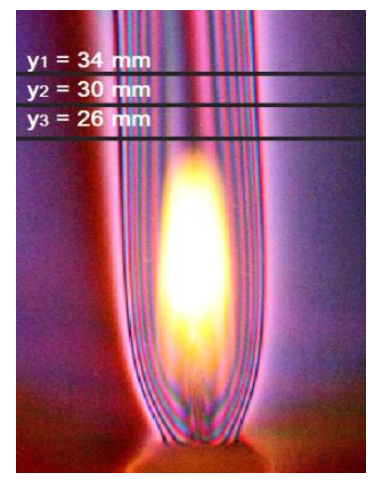

$a$

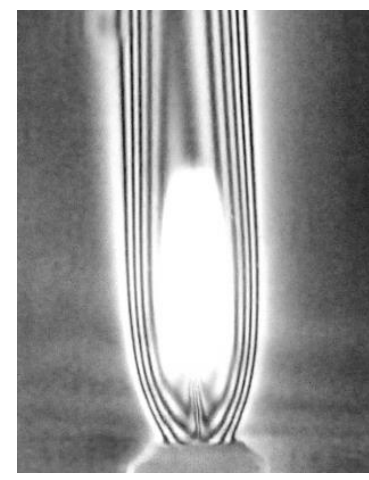

$b$

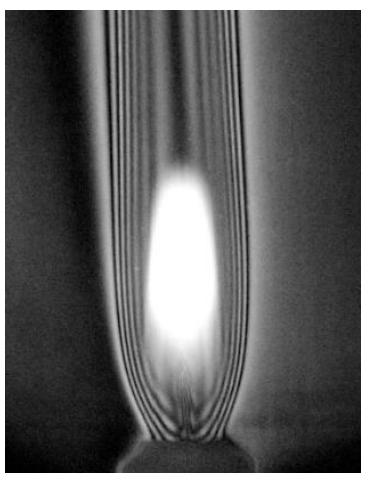

$c$

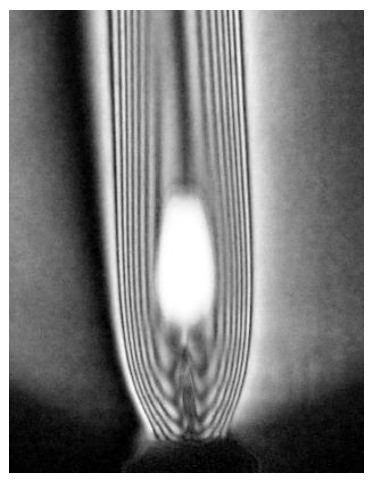

$d$

Figure 3: $(a)$ - experimental RGB-hilbertograms of the candle flame; $(b)-$ R-channel, $\lambda_{1}=636 \mathrm{~nm}$; (c) - G-channel, $\lambda_{2}=537 \mathrm{~nm}$; (d) - B-channel, $\lambda_{3}=466 \mathrm{~nm}$

Reconstruction of the radial distribution of the air flow temperature in the section at a distance of $y$ $=26 \mathrm{~mm}$ from the end of the candle wick for the R-channel is shown in figure 4 . The red line represents the phase function $\Delta \psi(r, y)$ obtained from the Abel equation (1), the green and black lines represent the interferogram and the hilbertogram, respectively, reconstructed from the phase function $\Delta \psi(r, y)$. The blue line is the experimental hilbertogram.

The method used consists in the iterative selection of the radial temperature profile represented by the Bezier curve (a special case of B-splines), followed by the calculation of the refractive index $n(r, y)$ and the phase function $\Delta \psi(r, y)$. Further, the hilbertogram (interferogram) is calculated from the phase function. The iterative algorithm is repeated until the points of the local minima of the Hilbert bands on the experimental and reconstructed hilbertograms coincide. The adequacy of the phase functions 
obtained from the solution of the Abel equation and the real one is confirmed by the coincidence of the reconstructed temperature fields in the selected areas.

In figure 5. $a$ shows the reconstructed radial temperature distributions of the air flow from the candle flame in the section $y=26 \mathrm{~mm}$ for three RGB channels. There are good matches. The distributions of the refractive index $n(r, y)$ in the section $y=26 \mathrm{~mm}$ for each of the three RGB channels are shown in figure. 5.b. In figure 5.c shows the corresponding distributions of the phase function $\Delta \psi(r, y)$ in the section $y=26 \mathrm{~mm}$, obtained from the solution of the Abel equation (1).
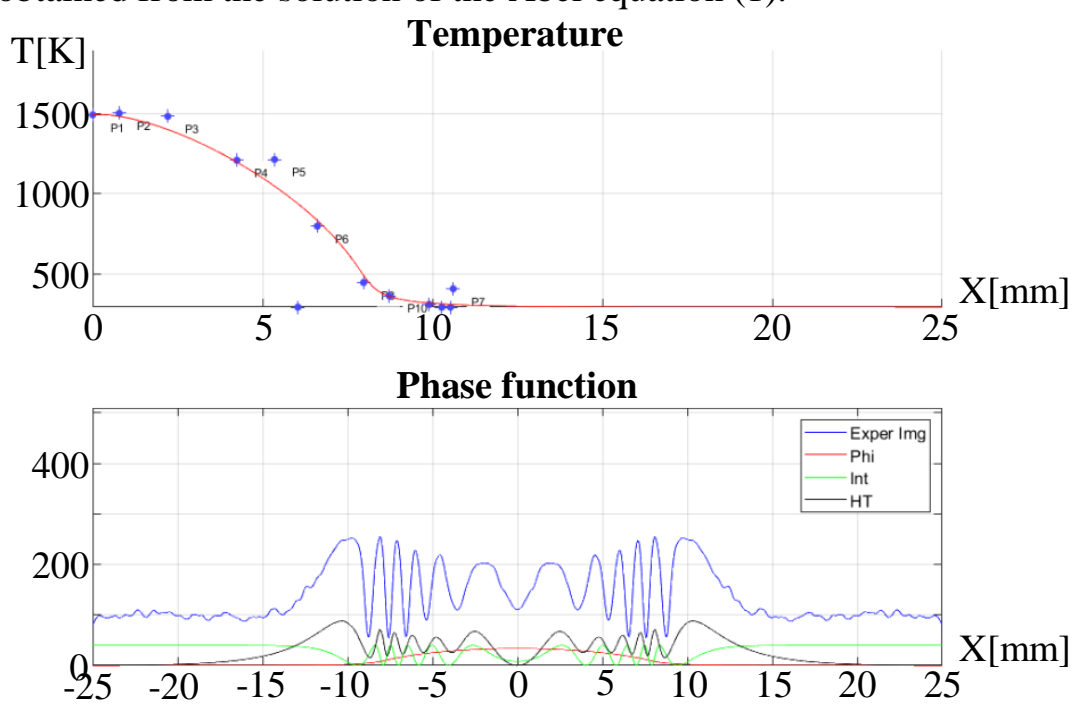

Figure 4: Reconstruction of the phase structure and temperature of the air flow from the candle flame in the section $y=26 \mathrm{~mm}$ : R-channel, $\lambda_{1}=636 \mathrm{~nm}$

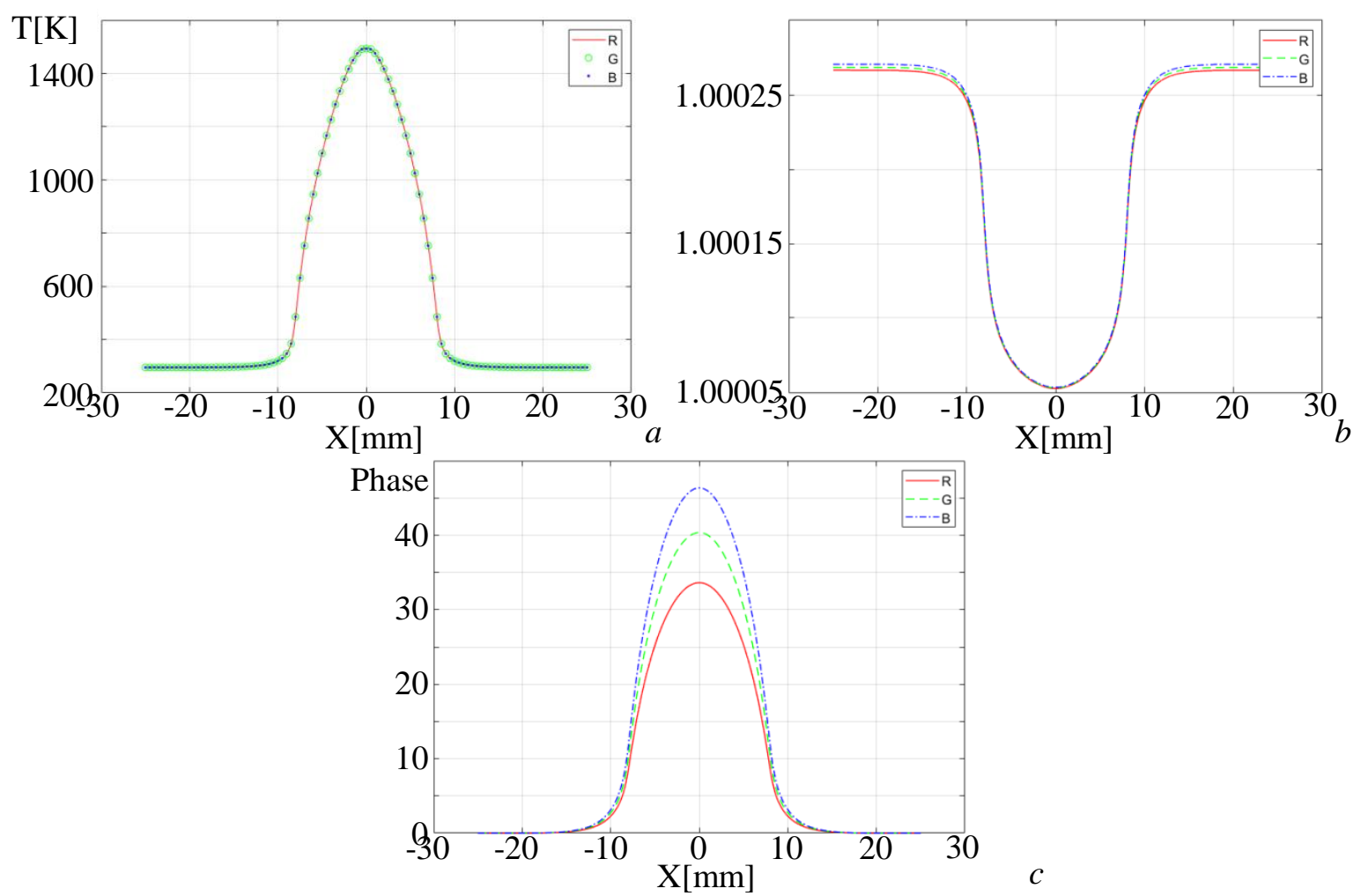

Figure 5: Section $y=26 \mathrm{~mm}:(a)$ - radial temperature distribution of the air flow from the candle flame for three RGB channels; $(b)$ - is the corresponding refractive index; $(c)$ - phase function

In figure 6 shows the temperature field of an axisymmetric section of the air flow heated by a candle flame, obtained using piecewise cubic Hermitian interpolation of the reconstructed temperature values in sections at heights $y=26,30$, and $34 \mathrm{~mm}$ (sections are shown in figure 3.a). 
Verification of the results obtained is confirmed by solving the direct problem: from the reconstructed temperature field (figure 6), the Hilbert images are reconstructed. They are compared with the images obtained in the experiment (figure 7). The experimentally obtained and reconstructed structures are of a similar nature. This confirms the reliability of the results. Some discrepancy is associated with the influence of dynamic perturbations of the air surrounding the flame on its axial symmetry in a real experiment.

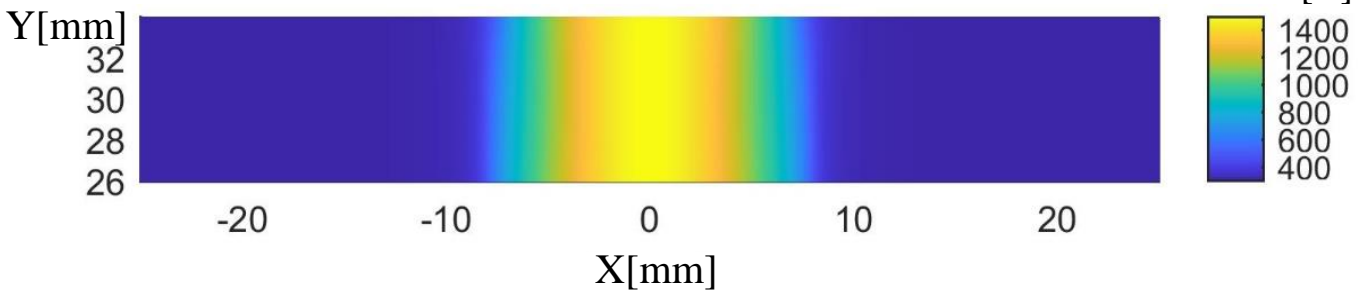

Figure 6: The temperature field of the heated air flow from the candle flame, reconstructed from RGBHilbert images
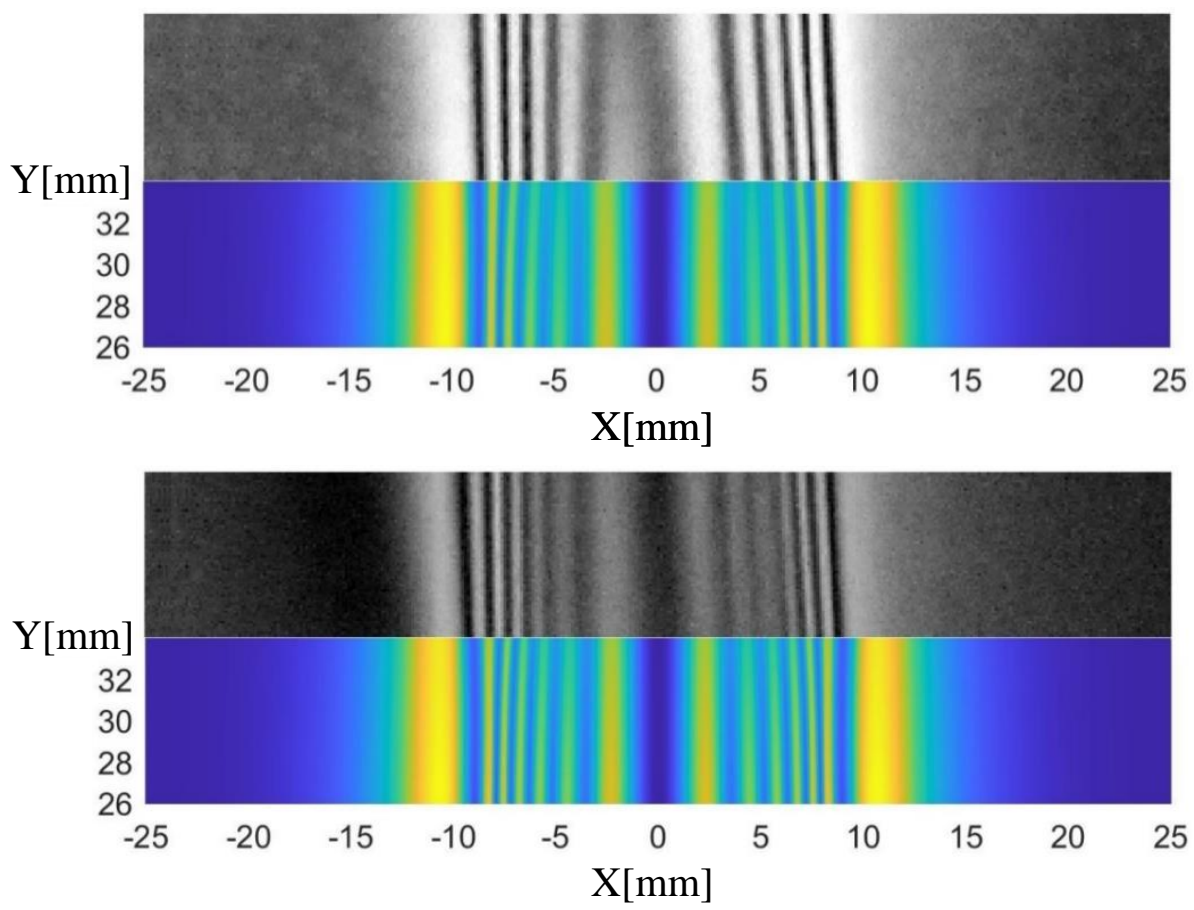

$a$

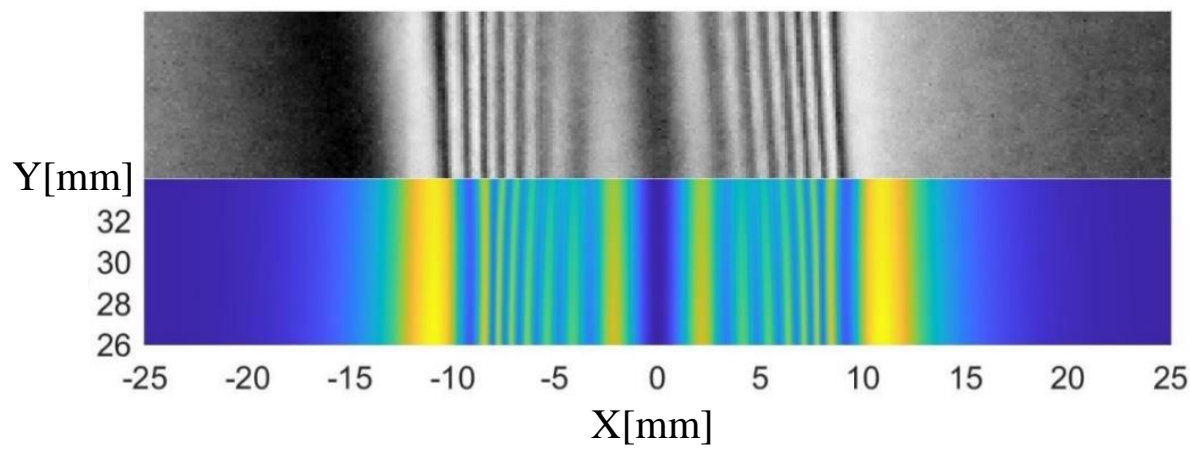

$b$

$c$

Figure 7: Comparison of experimental and simulated hilbertograms: $(a)-\mathrm{R}$-channel, $\lambda_{1}=636 \mathrm{~nm}$; (b) - G-channel, $\lambda_{2}=537 \mathrm{~nm}$; (c) - B-channel, $\lambda_{3}=466 \mathrm{~nm}$

\section{Reconstruction of the radial temperature profile in the structure of a hydrogen-air diffusion flame}

According to the dispersion formula of Gladstone-Dale [14, 15] 


$$
n_{k}(\lambda)=A_{k}\left(1+\frac{B_{k}}{\lambda^{2}}+\frac{D_{k}}{\lambda^{4}}\right),
$$

the refractive index of the $k$-th component of the burning mixture depends on the wavelength of the radiation source and parameters $A_{k}, B_{k}$, and $D_{k}$, which are determined empirically by measuring $n_{k}(\lambda)$ for three different values $\lambda$. When calculating by formula (3), we can restrict ourselves to a term with a quadratic dependence on the wavelength.

The refractive index of the entire mixture (hydrogen-air flame) is defined as

$$
n-1=\frac{p}{p_{\text {n.c. }}} \frac{T_{n . c .}}{T} \sum_{k} A_{k}\left(1+\frac{B_{k}}{\lambda^{2}}\right) \cdot C_{k},
$$

where $p$ is the pressure; $p_{n . c .}-$ atmospheric pressure under normal conditions (101 $\left.325 \mathrm{~Pa}\right) ; T$ temperature; $T_{n . c .}-$ temperature under normal conditions $\left(0^{\circ} \mathrm{C} \sim 273 \mathrm{~K}\right)$.

The method for reconstructing the temperature distribution from the measured values of the refractive index is based on equation (4), which relates $n$ to the temperature and composition of the gas mixture at a point. When hydrocarbons are burned in air, the refractive indices of the reacting components differ slightly from each other. A hydrogen flame, in contrast to a hydrocarbon flame, is characterized by a wide variety of partial optical properties of the gas mixture. In this case, an assessment of the content of the main components $\mathrm{H}_{2}, \mathrm{~N}_{2}, \mathrm{O}_{2}$ and $\mathrm{H}_{2} \mathrm{O}$ is required. The sufficiency of such a partial structure for determining the refractive index of a mixture was verified using experimental data [16]. It should be noted that when working with a hydrogen-oxygen flame, taking into account ionization can affect the accuracy of determining the temperature [17].

An image of the structure of a hydrogen-air flame visualized by Hilbert, obtained synchronously at three different wavelengths $\left(\lambda_{1}=636 \mathrm{~nm}, \lambda_{2}=537 \mathrm{~nm}\right.$, and $\left.\lambda_{3}=466 \mathrm{~nm}\right)$, is shown in figure 8.a, the division of the frame into RGB channels is shown in figure 8.b-8.d.

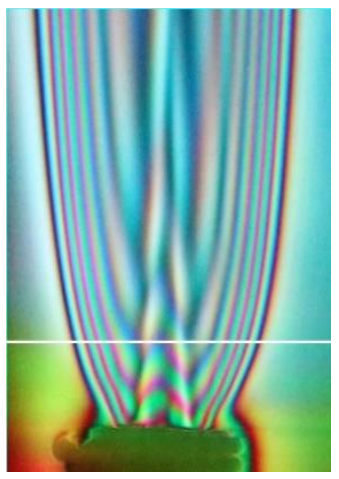

$a$

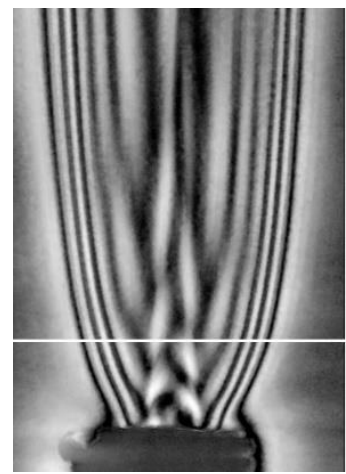

$b$

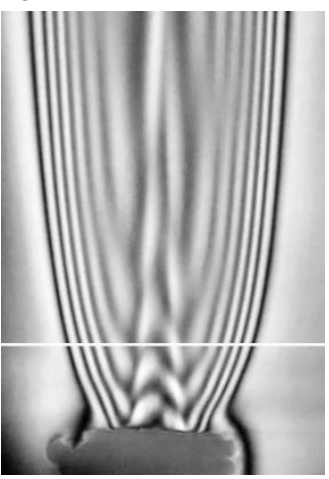

c

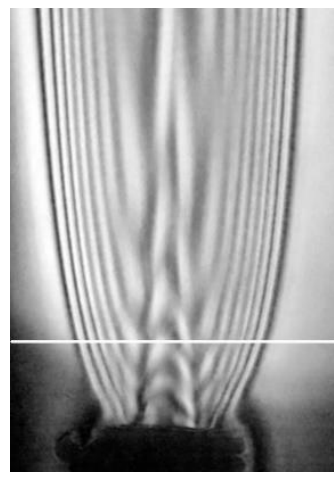

$d$

Figure 8: $(a)$ - RGB-hilbertogram of a hydrogen-air flame $\left(\mathrm{H}_{2} / \mathrm{N}_{2}\right.$, hydrogen volume fraction $\left.25 \%\right) ;(b)$ - R-channel, $\lambda_{1}=636 \mathrm{~nm}$; $(c)-$ G-channel, $\lambda_{2}=537 \mathrm{~nm}$; $(d)$ - B-channel, $\lambda_{3}=466 \mathrm{~nm}$; (white line -6 $\mathrm{mm}$ cross-section from the end of the burner tube)

For solve the inverse problem - to restore the flame temperature and molar fractions of fuel combustion products, it is necessary to restore the value of the phase function $\Delta \psi$ from the experimental hilbertogram and, having solved the Abel equation, determine the refractive index $n$ of the medium. Thus, by carrying out measurements at different wavelengths and using formulas (3)-(4), it is possible to restore the values of molar concentrations of fuel combustion products and, accordingly, the temperature distribution in the flame.

The area of combustion of a hydrogen-air flame can be conditionally divided into two zones, consisting of 3 main chemical elements of combustion products. Inner zone $-\mathrm{H}_{2}, \mathrm{H}_{2} \mathrm{O}$ and $\mathrm{N}_{2}$; outer zone $\mathrm{O}_{2}, \mathrm{H}_{2} \mathrm{O}$ and $\mathrm{N}_{2}$. The zone boundary is the area with the maximum temperature (flame front), since in its vicinity both the fuel and the oxidizer tend to zero. To determine $T$ and $C_{k}$ in each zone is sufficient to make measurements at three different wavelengths of the probing field.

In figure 9 is a graph illustrating the initial recovery of the phase function $\Delta \psi(r, y)$ at $6 \mathrm{~mm}$ from the end of the burner tube for the R-channel. The red curve represents the phase function $\Delta \psi(r, y)$, the blue curve is the section of the experimental hilbertogram, the black curve is the hilbertogram reconstructed from the phase function $\Delta \psi(r, y) .0 \mathrm{~mm}$ is the center of the burner tube.

The method for determining the phase function consisted in iterative sequential selection of the shape and height of the curve represented by the Bezier polynomial (a special case of B-splines), and 
the subsequent calculation of the refractive index $n(r, y)$. Further, according to the distribution $n(r, y)$ for the selected area, the hilbertogram is reconstructed, which is compared with the experimental hilbertogram. The iterative algorithm is repeated until the coordinates of the local extrema of the experimental and reconstructed hilbertograms coincide. The coincidence of the coordinates of the extrema of the experimental and reconstructed hilbertograms means checking the results obtained. When restoring the phase function $\Delta \psi(r, y)$, the values $A_{k}$ and $B_{k}$ were taken from the reference data.

\section{Phase function}

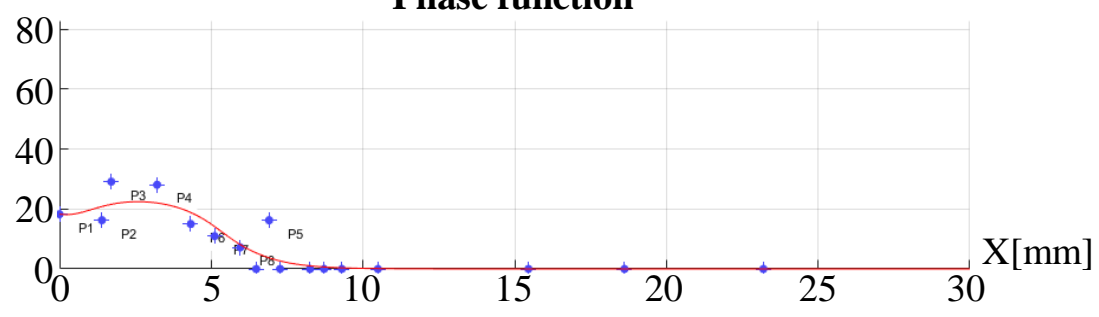

Hilbertogram

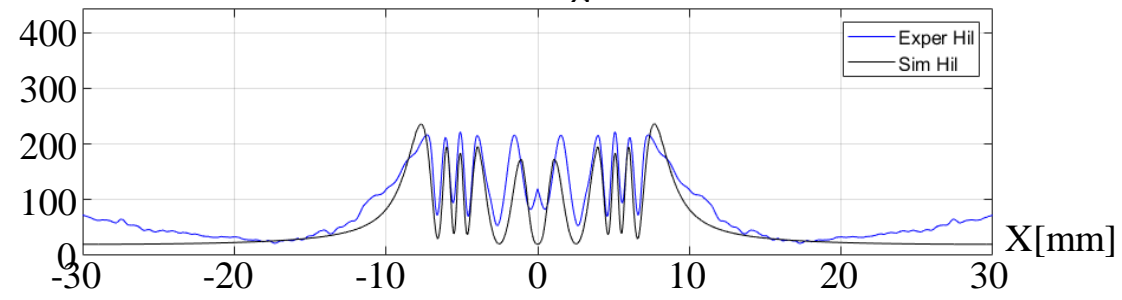

Figure 9: Experimental and reconstructed phase functions $\Delta \psi(r, y)$ and hilbertograms with a cross section of $6 \mathrm{~mm}$ : R-channel, $\lambda_{1}=636 \mathrm{~nm}$

During the experiment, the pressure in the room was equal to $100500 \mathrm{~Pa}$, the temperature was $24.8^{\circ} \mathrm{C}$, and the air humidity was $19 \%$.

In figure 10.a shows the values of the phase function $\Delta \psi(r, y)$ in a $6 \mathrm{~mm}$ section for three RGB channels. In figure 10.b shows the radial distributions of the refractive index $n(r, y)$, calculated from the obtained data $\Delta \psi(r, y)$.
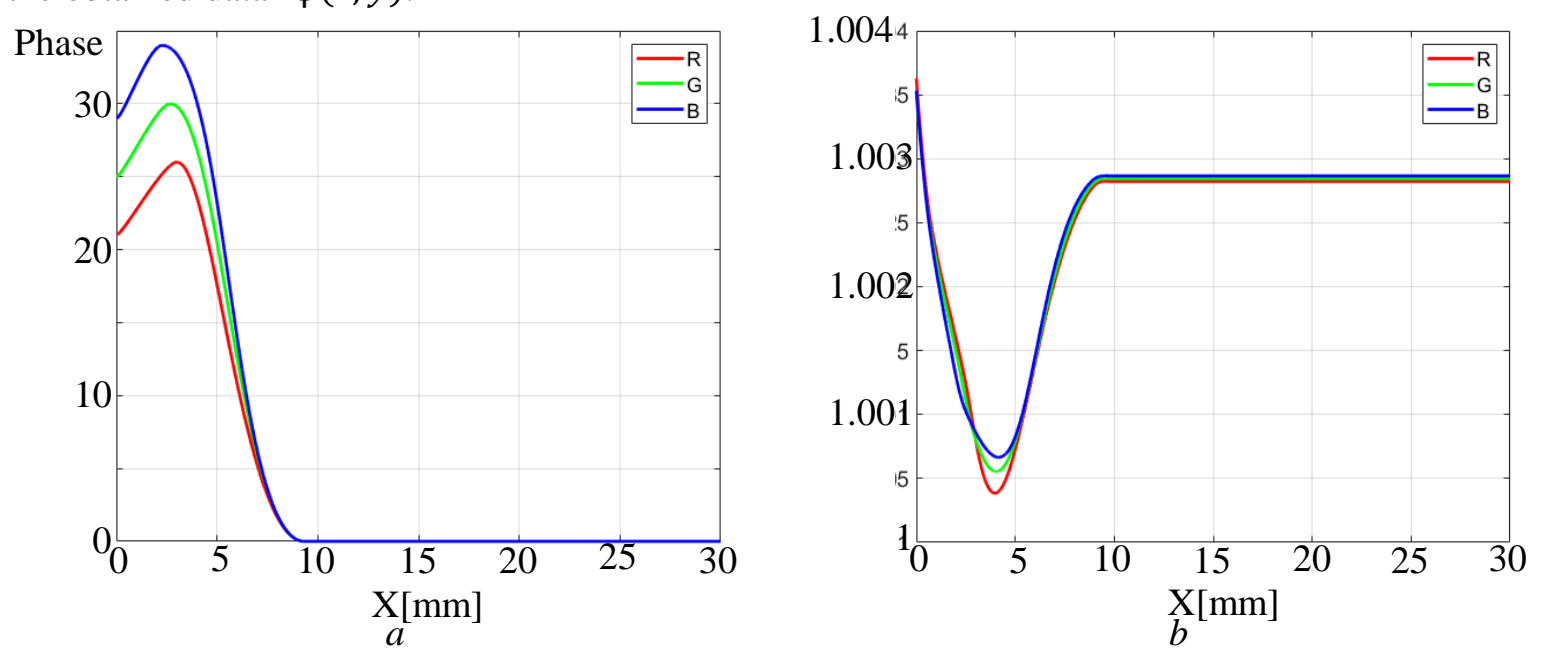

Figure 10: Section $6 \mathrm{~mm}$ : $(a)$ - reconstructed phase functions $\Delta \psi(r, y)$ for three RGB channels; $(b)-$ reconstructed radial profile of the refractive index $n(r, y)$ for three RGB channels

As a verification of the results obtained, the hilbertograms were calculated from the reconstructed phase function and compared with the hilbertograms obtained in the experiment (figure 11). Comparing the curves, we see that the points of the local minima of the Hilbert bands on the experimental and reconstructed hilbertograms coincide. This confirms the reliability of the approximation of the radial temperature field of a hydrogen-air flame (figure 12). 

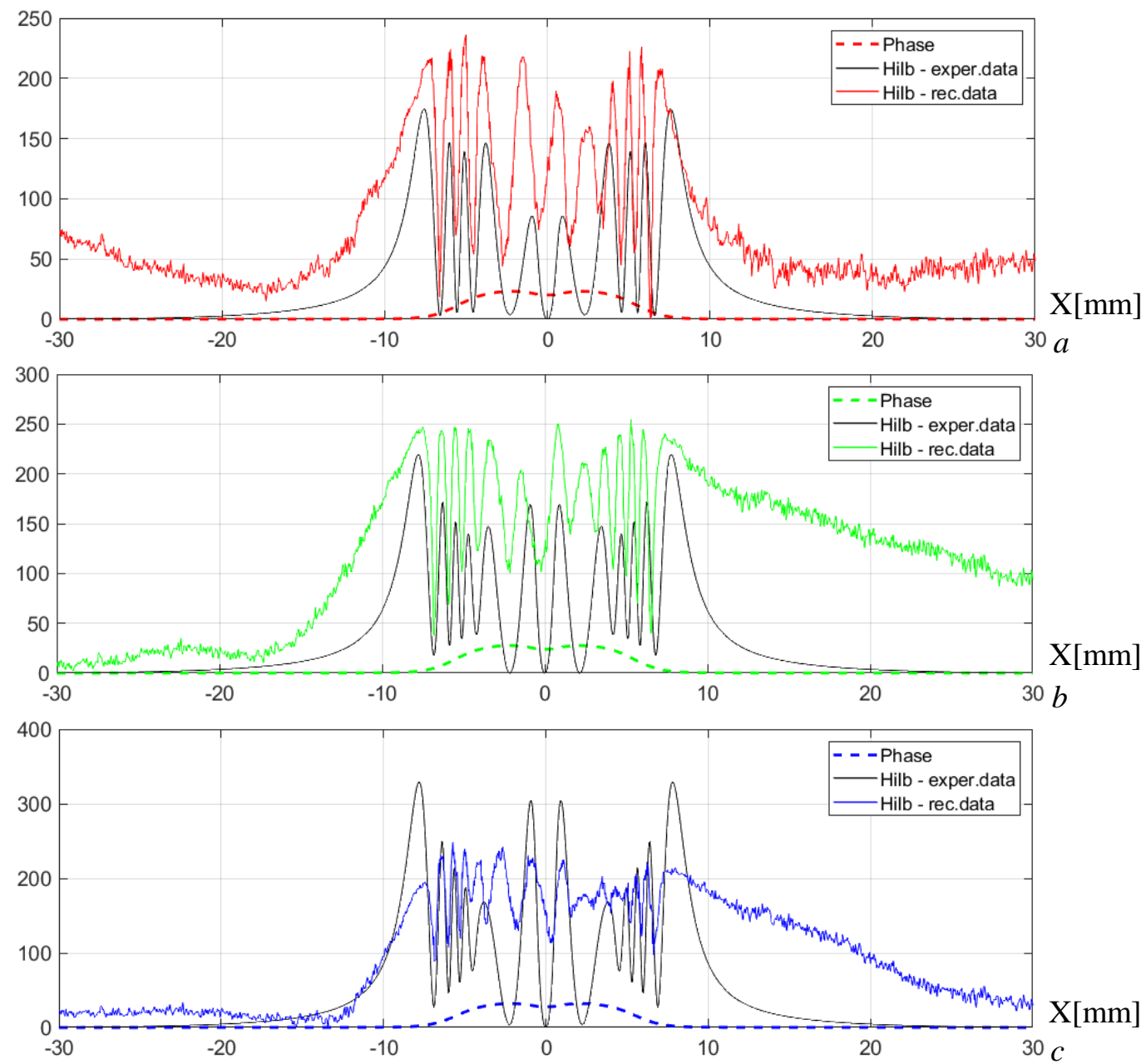

Figure 11: Comparison of experimental and reconstructed hilbertograms: $(a)-\mathrm{R}$-channel, $\lambda_{1}=636$ $\mathrm{nm}$; $(b)-\mathrm{G}$-channel, $\lambda_{2}=537 \mathrm{~nm}$; $(c)-$ B-channel, $\lambda_{3}=466 \mathrm{~nm}$; the solid line is the section of the experimental hilbertogram, the solid black line is the reconstructed hilbertogram, the dashed line is the reconstructed phase function $\Delta \psi(r, y)$

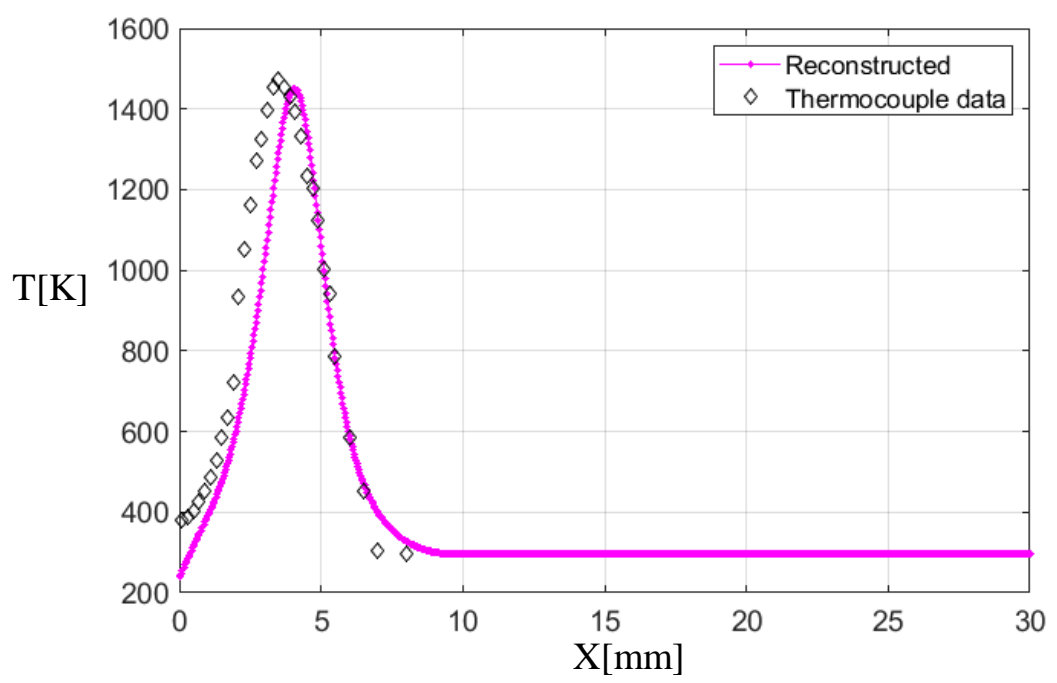

Figure 12: Reconstructed radial temperature field of an axisymmetric section of a hydrogen-air flame in a $6 \mathrm{~mm}$ section: solid line - reconstructed temperature $T$; black dots are the temperature measured by the thermocouple

The maximum temperature in the flame front is close to the adiabatic combustion temperature, which is $1490 \mathrm{~K}$ for an $\mathrm{H}_{2} / \mathrm{N}_{2}$ mixture (25\%) in air. The discrepancies in the experimental and reconstructed data are due to the violation of the axial symmetry of the flame in a real experiment due to the influence 
of dynamic disturbances of the air surrounding the flame. The difference between the reconstructed temperature values and the thermocouple measurements can also be introduced by the influence of the thermocouple on the flame or possible variability of the flame.

\section{Conclusion}

The method of the multiwave RGB-optical Hilbert diagnostics of phase and temperature fields in the approximation of axial symmetry of the object under study (in an air heat flow from a candle flame, hydrogen-air diffusion flame) has been theoretically and experimentally substantiated. The verification of the results is provided by comparing the hilbertograms obtained in the experiment and the hilbertograms reconstructed from phase structures using the Abel transform. One of the important advantages of the proposed method for flame diagnostics is the potential for measurements with a spatial resolution of up to several microns, which makes it possible to study the entire range of scales of the reacting flow up to Kolmogorov. The issue of visualization of asymmetric temperature fields is considered in perspective. The tomographic attachment will be added to the diagnostic complex in this case. This will allow visualization of the phase function in four different directions and estimate the flame temperature.

\section{Acknowledgements}

The work was carried out within the framework of the state assignment of IT SB RAS No. $121031800217-8$.

\section{References}

[1] J. Li, H. Huang, Y. Bai, S. Li, N. Kobayashi, Combustion and heat release characteristics of hydrogen/air diffusion flame on a micro-jet array burner, International Journal of Hydrogen Energy 43, No 29 (2018) 13563-13574. doi.org/10.1016/j.ijhydene.2018.04.195.

[2] T. S. Cheng, J.-Y. Chen, R. W. Pitz, Raman/LIPF data of temperature and species concentrations in swirling hydrogen jet diffusion flames: Conditional analysis and comparison to laminar flamelets, Combustion and Flame 186 (2017) 311-324.

[3] A. J. Smits, T. T. Lim, Flow visualization: techniques and example, London: Imperial College Press, 2010.

[4] Y. Yong, Q. Tian, L. Gang, M. M. Hossain, G. Gilabert, L. Shi, Recent Advances in Flame Tomography, Chinese Journal of Chemical Engineering 20, No 2 (2012) 389-399. doi: 10.1016/S1004-9541(12)60402-9

[5] Qunxing Huang, Fei Wang, Jianhua Yan and Yong Chi, Simultaneous estimation of the 3-d soot temperature and volume faction distributions in asymmetric flames using high-speed stereoscopics images, Applied Optics 51, No 15 (2012) 2968-2978. doi.org/10.1364/AO.51.002968

[6] J. A. H. Dreyer, R. I. Slavchov, E. J. Rees et al., Improved methodology for performing the inverse Abel transform of flame images for cilir ratio pyrometry, Applied Optics 58, No 10 (2019) 26622670. doi: 10.1364/AO.58.002662

[7] P. P. Khramtsov, O. G. Penyazkov, I. N. Shatan, Temperature measurements in an axisymmetric methane-air flame using Talbot images, Experiments in Fluids 56 (2015). doi: 10.1007/s00348015-1906-x

[8] V. A. Arbuzov, E. V. Arbuzov, Yu. N. Dubnishchev, O. S. Zolotukhina, V. V. Lukashov, Recovery of the temperature field in the case of combustion of premixed propane-air mixtures by methods of the Hilbert optics, Optoelectronics, Instrumentation and Data Processing 56, No 1 (2020) 6167. doi: $10.3103 / \mathrm{s} 8756699020010082$

[9] L. A. Vasiliev, Shadow methods, Publishing house "Science", Main edition of physical and mathematical literature, Moscow, 1968. (in Russian) 
[10] Yu. N. Dubnishchev, V. A. Arbuzov, E. V. Arbuzov, O. S. Zolotukhina, V. V. Lukashov, Optical diagnostics of temperature field of an axisymmetric flame, Scientific Visualization 11, No 4 (2019) 130-139. doi: 10.26583/sv.11.4.11

[11] Yu. N. Dubnishchev, Theory and conversion of signals in optical systems, SPb. Lan Publishing House, 2011. (in Russian)

[12] Yu. N. Dubnishchev, V. A. Arbuzov, E. V. Arbuzov, O. S. Zolotukhina, V. V. Lukashov, Reconstruction of the spatial phase and temperature structure of the candle flame by the methods of Hilbert optics and shear interferometry, in: GraphiCon'2019 Proceedings, Vol. 1, Bryansk, September 23-26, 2019, p. 4. doi: 10.30987/graphicon-2019-1-58-61.

[13] Ashrafi Z. Najafian, M. Ashjaee, M. H. Askari, Two-dimensional temperature field measurement of a premixed methane/air flame using Mach-Zehnder interferometry, Optics Communications 341 (2015) 55-63. doi: 10.1016/j.optcom.2014.12.004

[14] V. Hauf, U. Grigul, Optical methods in heat transfer, Mir, Moscow, 1973. (in Russian)

[15] B. V. Ioffe, Refractometric methods of chemistry, 3rd ed., Rev. L.: Chemistry, Leningrad. separation, 1983. (in Russian)

[16] V. V. Toro, A. V. Mokhov, H. B. Levinsky, M. D. Smooke, Combined experimental and computational study of laminar, axisymmetric hydrogen-air diffusion flames, Proceedings of the Combustion Institute 30 (2005) 485-492. doi: 10.1016/j.proci.2004.08.221

[17] C. Yun-yun, Li Zhen-hua, S. Yang, H. An-zhi, Extension of the Gladstone-Dale equation for flame flow field diagnosis by optical computerized tomography, Applied Optics 48, No 13 (2009) 24852490. doi.org/10.1364/AO.48.002485 